\title{
Serum neurofilament light chain levels as a marker of upper motor neuron degeneration in patients with Amyotrophic Lateral Sclerosis
}

B. Gille*,† (D), M. De Schaepdryver*,‡, J. Goossens*, L. Dedeene*,‡, J. De Vocht§,ף, E. Oldoni**,

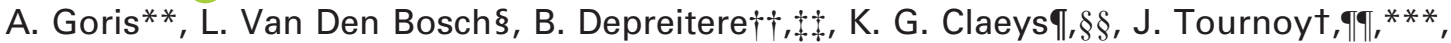
P. Van Damme $\S, \|^{1}{ }^{1}$ and K. Poesen ${ }^{*},{ }^{\prime}{ }^{1}$

*Laboratory for Molecular Neurobiomarker Research, Department of Neurosciences, KU Leuven, †Department of Chronic Disease, Metabolism and Ageing, KU Leuven, łLaboratory Medicine, University Hospitals Leuven, \$Laboratory of Neurobiology, Department of Neurosciences, KU Leuven and Center for Brain \& Disease Research VIB Leuven, ФDepartment of Neurology, Neuromuscular Reference Centre, University Hospitals Leuven, ${ }^{* *}$ Laboratory for Neuroimmunology, Department of Neurosciences, KU Leuven, t†Department of Neurosurgery, Neuromuscular Reference Centre, University Hospitals Leuven, $+\neq$ Research Group Experimental Neurosurgery and Neuroanatomy, KU Leuven, §§Laboratory for Muscle diseases and Neuropathies, Department of Neurosciences, KU Leuven, q9|Alzheimer Research Centre KU Leuven, Leuven Institute of Neuroscience and Disease and ***Department of Geriatric Medicine, University Hospitals Leuven, Leuven, Belgium

B. Gille, M. De Schaepdryver, J. Goossens, L. Dedeene, J. De Vocht, E. Oldoni, A. Goris, L. Van Den Bosch,

B. Depreitere, K. G. Claeys, J. Tournoy, P. Van Damme and K. Poesen (2018) Neuropathology and Applied Neurobiology

\section{Serum neurofilament light chain levels as a marker of upper motor neuron degeneration in patients with Amyotrophic Lateral Sclerosis}

Aims: Amyotrophic lateral sclerosis (ALS) is the most common motor neuron degeneration disease with a diagnostic delay of about 1 year after symptoms onset. In ALS, blood neurofilament light chain (NfL) levels are elevated, but it is not entirely clear what drives this increase and what the diagnostic performance of serum NfL is in terms of predictive values and likelihood ratios. The aims of this study were to further explore the prognostic and diagnostic performances of serum NfL to discriminate between patients with ALS and ALS mimics, and to investigate the relationship between serum NfL with motor neuron degeneration. Methods: The diagnostic performances of serum NfL were based on a cohort of 149 serum samples of patients with ALS, 19 serum samples of patients with a disease mimicking ALS and 82 serum samples of disease control patients. The serum NfL levels were correlated with the number of regions (thoracic, bulbar, upper limb and lower limb) displaying upper and/or lower motor neuron degeneration. The prognostic performances of serum NfL were investigated based on a Cox regression analysis. Results: The associated predictive values and likelihood ratio to discriminate patients with ALS and ALS mimics were established. Serum NfL was associated with motor neuron degeneration driven by upper motor neuron (UMN) degeneration and was independently associated with survival in patients with ALS. Conclusions: Altogether, these findings suggest that elevated serum NfL levels in ALS are driven by UMN degeneration and the disease progression rate and are independently associated with survival at time of diagnosis.

Keywords: amyotrophic lateral sclerosis, neurofilament light chain, motor neuron degeneration

Correspondence: Koen Poesen, UZ Leuven, Herestraat 49, 3000 Leuven, Belgium. Tel: +32 163470 13; Fax: +32 16347010 ; E-mail: koen.poesen@uzleuven.be

${ }^{1}$ Shared last authorship. 


\section{Introduction}

Lou Gehrig's disease or amyotrophic lateral sclerosis (ALS) is characterized by a degeneration of upper motor neurons (UMN) in the motor cortex and lower motor neurons (LMN) in the brain stem and spinal cord, resulting in a progressive muscle weakness [1,2]. The diagnosis of ALS is currently supported by a clinical examination and an electromyography (EMG), requiring the degeneration of both UMN and LMN, the evidence of disease spreading to other regions and the exclusion of another aetiology [1,3-5]. The diagnosis of ALS is made on average 10-12 months after onset of first symptoms, when patients are already well advanced in their ALS disease trajectory [6]. Thus, an earlier diagnosis of ALS is desirable for early initiation of therapy and for inclusion of patients in clinical trials. Diagnostic biomarkers for ALS in early disease stages might shorten the diagnostic delay. We and others showed that the cerebrospinal fluid (CSF) levels of neurofilament light chain (NfL) and phosphorylated neurofilament heavy chain $(\mathrm{pNfH})$ in patients with ALS are significantly higher in comparison with the CSF levels of ALS mimics, displaying good predictive values towards the diagnosis at the early or conventional diagnostic stage [6-9]. Furthermore, we showed that CSF NfL and pNfH levels correlated with the extent of UMN and LMN degeneration [6,7]. In line with these findings, we and others described that the serum concentrations of pNfH in patients with ALS were higher than in patients with an ALS mimic at the conventional diagnostic stage [7]. A similar change for NfL in blood of patients with ALS has recently been reported [8]. Nevertheless, we did not observe any correlation between pNfH in blood with UMN or LMN loss in ALS, matching our finding of $\mathrm{pNfH}$ in blood being less performant than pNfH in CSF to discriminate between ALS and ALS mimics [7]. It is unclear yet to what extent NfL in blood of patients with ALS correlates with UMN and/or LMN degeneration, and if the raised levels of NfL in blood are useful to discriminate between patients with ALS and ALS mimics in terms of predictive values and likelihood ratios.

The objective of this study was to evaluate the diagnostic performances of NfL in blood and whether NfL levels in blood correlate with the hallmark features of ALS, such as the extent of UMN and LMN degeneration, the disease progression rate and survival.

\section{Materials and methods}

We sampled peripheral blood in 5-ml BD Vacutainer ${ }^{\circledR}$ serum separation tubes (catalogue \#:367955; Becton Dickinson and Company, Franklin Lakes, NJ, USA) of patients referred to the University hospital of Leuven neuromuscular reference centre (NMRC) with complaints suspicious of ALS (including muscle weakness or speech/swallowing/breathing difficulties), and hence with a differential diagnosis including ALS, between April 2014 and August 2017. All participants signed an informed consent. Patients were followed up and eventually received the diagnosis of (i) ALS $(n=149)$ (indicating $56 \%$ of patients diagnosed at the NMRC at time of study inclusion) or of (ii) an ALS mimic ( $n=19)$, including patients presenting with symptoms reminiscent of ALS but for whom ALS was ruled out upon follow-up by an expert neurologist at the NMRC (PVD, KGC). The diagnosis of ALS was based on the Awaji and revised El Escorial criteria [3,5,10] (Table 1). Among those patients, 15 were C9orf72 mutation carriers and 15 were diagnosed with frontotemporal dementia (FTD). In addition, 11 patients were diagnosed with primary lateral sclerosis (PLS) and six patients were diagnosed with progressive muscular atrophy (PMA) (Table 2). Moreover, we also included patients whose blood was sampled at the diagnostic phase and who eventually received a diagnosis of Guillain-Barré syndrome (GBS), chronic inflammatory demyelinating polyneuropathy (CIDP) or hereditary spastic paraplegia (HSP) by an expert neurologist at the NMRC.

Symptom duration in patients with ALS was defined as the time difference between estimated first symptom onset (muscle weakness or speech/swallowing/ breathing difficulties) and blood sampling. The disease progression rate, expressed in points/month, was calculated as the difference between the maximum score of 48 and the ALS functional rating scale revised (ALSFRS-R) score the closest to blood sampling, divided by the symptom duration (in months) at date of ALSFRS-R score. Hundred and twenty-four patients with ALS with a delay between blood sampling and ALSFRS-R scoring within 3 months (median: 0.1 month; range: 0-3 months) were included in the correlation analysis between the serum NfL levels and the disease progression rate. Patients with a disease 
Table 1. Clinical characteristics of the patients with ALS

\begin{tabular}{|c|c|}
\hline & Median (IQR; range) \\
\hline$n(\mathrm{~m} / \mathrm{f})$ & $149(94 / 55)$ \\
\hline Age at blood sampling (years) & $62(15.4 ; 29-93)$ \\
\hline $\begin{array}{l}\text { Age at estimated symptom onset } \\
\text { (years) } \dagger\end{array}$ & $60(14.8 ; 25-84)$ \\
\hline $\begin{array}{l}\text { Site of onset (bulbar/spinal/ } \\
\text { generalized/unknown) } \dagger\end{array}$ & $34 / 105 / 4 / 6$ \\
\hline Diagnostic delay (months) $\ddagger$ & $10.6(8.6 ; 3-107)$ \\
\hline $\begin{array}{l}\text { Symptoms duration at blood } \\
\text { sampling (months) } \dagger\end{array}$ & $13.7(12.5 ; 2-240)$ \\
\hline $\begin{array}{l}\text { ALSFRS-R score closest to date of } \\
\text { blood sampling } \$\end{array}$ & $37(12.0 ; 11-47)$ \\
\hline $\begin{array}{l}\text { Disease progression rate (points/ } \\
\text { month) }\end{array}$ & $0.672(0.75 ; 0.058-5.00)$ \\
\hline FVC $(\%) \dagger \dagger$ & $92(32.5 ; 25-142)$ \\
\hline Serum NfL (pg/ml) & $179(164.6 ; 0.3-1141)$ \\
\hline \multicolumn{2}{|l|}{ Serum NfL (pg/ml) } \\
\hline $\begin{array}{l}1 \text { Region with UMN } \\
\text { neurodegeneration }(n=14)\end{array}$ & $106(145 ; 25-370)$ \\
\hline $\begin{array}{l}2 \text { Regions with UMN } \\
\text { neurodegeneration }(n=31)\end{array}$ & $188(136 ; 12-768)$ \\
\hline $\begin{array}{l}3 \text { Regions with UMN } \\
\text { neurodegeneration }(n=55)\end{array}$ & $251(155 ; 62-1141)$ \\
\hline \multicolumn{2}{|l|}{ Serum NfL $(\mathrm{pg} / \mathrm{ml})$} \\
\hline $\begin{array}{l}1 \text { Region with LMN } \\
\text { neurodegeneration }(n=33)\end{array}$ & $191(169.2 ; 12-781)$ \\
\hline $\begin{array}{l}2 \text { Regions with LMN } \\
\text { neurodegeneration }(n=21)\end{array}$ & $221(281.7 ; 41-694)$ \\
\hline $\begin{array}{l}3 \text { Regions with LMN } \\
\text { neurodegeneration }(n=46)\end{array}$ & $225(161.4 ; 38-1141)$ \\
\hline \multicolumn{2}{|l|}{ Serum NfL $(\mathrm{pg} / \mathrm{ml})$} \\
\hline $\begin{array}{l}1 \text { Region with LMN and UMN } \\
\text { neurodegeneration }(n=34)\end{array}$ & $119(175.9 ; 12-781)$ \\
\hline $\begin{array}{l}2 \text { Regions with LMN and UMN } \\
\text { neurodegeneration }(n=47)\end{array}$ & $232(168.9 ; 41-768)$ \\
\hline $\begin{array}{l}3 \text { Regions with LMN and UMN } \\
\text { neurodegeneration }(n=19)\end{array}$ & 268 (192.6; 110-1141) \\
\hline \multicolumn{2}{|l|}{ Serum NfL (pg/ml) } \\
\hline $\begin{array}{l}\text { Slow disease progression rate } \\
(n=33)\end{array}$ & $112(81.4 ; 0.3-295)$ \\
\hline $\begin{array}{l}\text { Intermediate disease progression } \\
\text { rate }(n=61)\end{array}$ & $188(152.9 ; 12-554)$ \\
\hline $\begin{array}{l}\text { Fast disease progression rate } \\
(n=30)\end{array}$ & $255(215.1 ; 62-1141)$ \\
\hline
\end{tabular}

progression rate below the 25 th percentile $(<0.372)$ were considered as slow progressors, patients with a disease progression rate above the 75 th percentile $(>1.121)$ were considered as fast progressors and patients with a disease progression rate between or equal to 0.372 and 1.121 were identified as intermediate progressors. The number of regions affected by UMN degeneration was assessed clinically in the bulbar, upper limb and lower limb regions, according to
Table 1. (Continued)

\begin{tabular}{lc}
\hline & Median (IQR; range) \\
\hline Mean survival after sampling in months $(95 \% \mathrm{CI})$ \\
Low tertile NfL $(<115 \mathrm{pg} / \mathrm{ml})$ & $40.1(37.36-42.8)$ \\
Mid-tertile NfL $(115 \mathrm{pg} /$ & $23.6(19.78-27.44)$ \\
$\mathrm{ml} \leq x \leq 235 \mathrm{pg} / \mathrm{ml})$ & \\
High tertile NfL $(>235 \mathrm{pg} / \mathrm{ml})$ & $17.3(13.41-21.1)$ \\
\hline
\end{tabular}

95\% CI, 95th percentile confidence interval; ALS, amyotrophic lateral sclerosis; ALSFRS-R, ALS functional rating scale revised score; IQR, interquartile range; NfL, neurofilament light chain; FVC, forced vital capacity; LMN, lower motor neuron; UMN, upper motor neuron

$\dagger$ Six patients with unknown estimated date of symptoms onset or site of onset.

†Diagnostic delay could not be calculated in six patients who had an unknown date of symptoms onset and one patient had an unknown date of diagnosis.

§ALSFRS-R was not performed in seven patients.

"Eight patients without disease progression rate because ALSFRS$\mathrm{R}$ not performed in seven patients and one patient had an unknown date of symptoms onset.

$\dagger \dagger F V C$ was unavailable for 17 patients.

the Awaji and revised El Escorial criteria. UMN involvement in the thoracic region is not assessed routinely at our NMRC. The number of regions with LMN degeneration was assessed clinically and by EMG in the bulbar and thoracic regions and in the upper and lower limbs according to the revised El Escorial and Awaji criteria. One hundred patients with ALS with a maximum delay of 3 months between EMG or clinical assessment and blood sampling were included in the correlation between serum NfL and the number of regions affected by neurodegeneration. The forced vital capacity (FVC) score the closest to blood take was estimated with spirometry in 132 patients with ALS. A total of 113 patients with ALS having a possible minimum 1-year survival follow-up after blood sampling and measurement were included in the survival analysis (excluded sampling between December 2016 and 2017). Patients with ALS with missing data or a delay between blood sampling and ALSRFS-R, EMG or FVC assessment greater than 3 months were excluded from the multivariate Cox regression analysis. The CSF levels of NfL were assessed with an enzyme-linked immunosorbent kit (UmanDiagnostics, Umea, Sweden) as previously published [6]. The serum NfL levels were quantified with an electrochemiluminescent assay as previously described [11]. Patients were then stratified in tertiles according to their serum NfL levels: (i) lowest tertile 
Table 2. The serum levels of NfL in ALS mimics and controls

\begin{tabular}{|c|c|c|c|c|}
\hline Cohort group & Diagnosis & $n(\mathrm{~m} / \mathrm{f})$ & Age (years) & Serum NfL $(\mathrm{pg} / \mathrm{ml})$ \\
\hline \multirow[t]{11}{*}{ ALS mimics } & Chronic radiculopathy/polyradiculopathy & $7(6 / 1)$ & $54(6 ; 38-71)$ & $29(71 ; 21-152)$ \\
\hline & Brachial plexopathy & $2(2 / 0)$ & $44(7 ; 37-51)$ & $537(516 ; 20-1053)$ \\
\hline & Myasthenia Gravis & $2(1 / 1)$ & $73(1 ; 72-74)$ & $80(13 ; 67-93)$ \\
\hline & Cervical stenosis & $1(1 / 0)$ & 63 & 61 \\
\hline & Fewdon syndrome [12] & $1(0 / 1)$ & 25 & 21 \\
\hline & Hirayama syndrome & $1(1 / 0)$ & 23 & 6 \\
\hline & $\mathrm{IBM}$ & $1(1 / 0)$ & 80 & 74 \\
\hline & Suspected AMAN & $1(1 / 0)$ & 71 & 42 \\
\hline & MMN & $1(0 / 1)$ & 28 & 15 \\
\hline & Motor neuropathy & $1(0 / 1)$ & 57 & 20 \\
\hline & Brainstem vascular lesions & $1(0 / 1)$ & 72 & 19 \\
\hline \multirow[t]{3}{*}{ Disease controls } & GBS & $48(26 / 22)$ & $53(30.8 ; 7-86)$ & $123(280.6 ; 22-9045)$ \\
\hline & CIDP & $20(17 / 3)$ & $59(12.5 ; 31-75)$ & $101(117.5 ; 29-2863)$ \\
\hline & HSP & $14(8 / 6)$ & $46(22.2 ; 25-84)$ & 37 (32.3; 8-639) \\
\hline
\end{tabular}

Data expressed as the median (IQR; range).

ALS, amyotrophic lateral sclerosis; AMAN, acute motor axonal neuropathy; CIDP, chronic inflammatory demyelinating polyneuropathy; GBS, Guillain-Barré syndrome; HSP, hereditary spastic paraplegia; IBM, inclusion body myositis; IQR, interquartile range; MMN, multifocal motor neuropathy; Nfl, neurofilament light chain.

serum NfL $<115 \mathrm{pg} / \mathrm{ml}$; (ii) mid-tertile serum between or equal to $115 \mathrm{pg} / \mathrm{ml}$ and $235 \mathrm{pg} / \mathrm{ml}$ and (iii) highest tertile serum NfL $>235 \mathrm{pg} / \mathrm{ml}$. Longitudinal serum samples of 16 patients with ALS were available (mean follow-up $=15.5$ months; $\quad$ range $=3.8-32.0$ months). The different time points for a given patient were assessed on the same run to avoid analytical deviation.

\section{Statistical analysis}

Normality of the data and residuals were assessed with the D'Agostino \& Pearson's test. Correlations between the serum levels of NfL with the CSF levels NfL, symptom duration at blood sampling or the disease progression rate were assessed with the Spearman's rank-order correlation, with a spearman's coefficient correlation (Spearman's $\rho$ ) at a significance level of 5\%. Comparisons between the serum levels of NfL with the disease progression rate, the extent of motor neuron degeneration in patients with ALS or with the different diagnosis were assessed with the Kruskal-Wallis test corrected for multiple comparisons with the post hoc Dunn's test, at a significance level of 5\%. Logarithmic transformation of the serum levels of NfL limited the right skewed distribution of the serum NfL levels, allowing the analysis of covariance. Optimal receiver operating characteristics (ROC) curves were generated to calculate the area under the curve (AUC) and the highest Youden's index was used to estimate the optimal cut-offs for which sensitivity, specificity, positive and negative likelihood ratio (+LR and -LR, respectively) and positive and negative predictive values (PPV and NPV, respectively) were estimated. For the comparison between slow and fast progressors the predictive values were calculated based on the prevalence obtained by Gomeni \& Fava [13]. For the comparison between ALS and ALS mimics, the predictive values were calculated based on the prevalence of ALS and ALS mimics in our data set. The effect of age and gender on the AUC was assessed in a multivariate model using logistic regression and an adjusted AUC was reported, based on the predictive probabilities, in the case of significant differences with the AUC obtained with univariate model using the serum NfL values. The Cox proportional-hazard regression analysis was performed via the backward method with a $P$-value threshold of 0.157 , with the time to event being the time from blood sampling to death [14]. Based on a recent study of prognostic factors in ALS [15], the following parameters were included as survival variables into the multivariate Cox regression: the tertiles of the serum levels of NfL, the disease progression rate stratified as slow, intermediate and fast, the number of regions with UMN and LMN degeneration, age at blood sampling, the FVC, gender, the diagnostic delay, the C9orf72 status, FTD status and the site of onset. The exclusion criteria for the Cox regression were a follow-up shorter than a year (sampling between December 2016 and 2017) and, for the multivariate 
Cox regression, a time difference among the date of ALSFRS-R, EMG and FVC with the date of blood sampling greater than 3 months. The Kaplan-Meier analysis was performed only on the tertiles of the serum levels of NfL and on the full data set $(n=149)$. Statistical testing was performed with GraphPad Prism (v.7.01; GraphPad Software Inc, San Diego, CA, USA), Kaplan-Meier, ROC curve, logistic regression and analysis of covariance were performed in MedCalc (v.17.9; MedCalc Software bvba, Ostend, Belgium) and univariate and multivariate Cox proportional-hazard regressions were performed in SPSS version 25 (SPSS, Chicago, IL, USA). $\quad * P<0.05, \quad * * P<0.01$, $* * * P<0.001$ and $* * * * P<0.0001$ were considered significant.

\section{Results}

The demographics of the patients with ALS and the different control cohorts are listed in Tables 1 and 2 respectively. Serum NfL strongly correlated with the CSF NfL as assessed on 97 paired samples of patients with ALS (Spearman's $\rho=0.79, \quad P<0.0001$ ) (Figure 1a). The median time differences between blood sampling and lumbar puncture was less than 24 h (95 pairs of 97 were sampled on the same day).

\section{Serum NfL are correlated with symptom duration and the disease progression rate}

In serum of patients with ALS, NfL correlated negatively with symptom duration at time of blood sampling (Spearman's $\rho=-0.39, P<0.0001$ ) (Figure 1b). The median symptom duration to blood sampling was 13.7 months [interquartile range $(\mathrm{IQR})=12.5$ months; $\quad$ range $=2$ 240 months]. Fast progressors and slow progressors displayed a short and long symptom duration at blood sampling respectively (median symptom duration fast progressors $=7.3$ months; $\quad \mathrm{IQR}=4.7$ months; range $=$ 3.4-31.0 months, median symptom duration slow
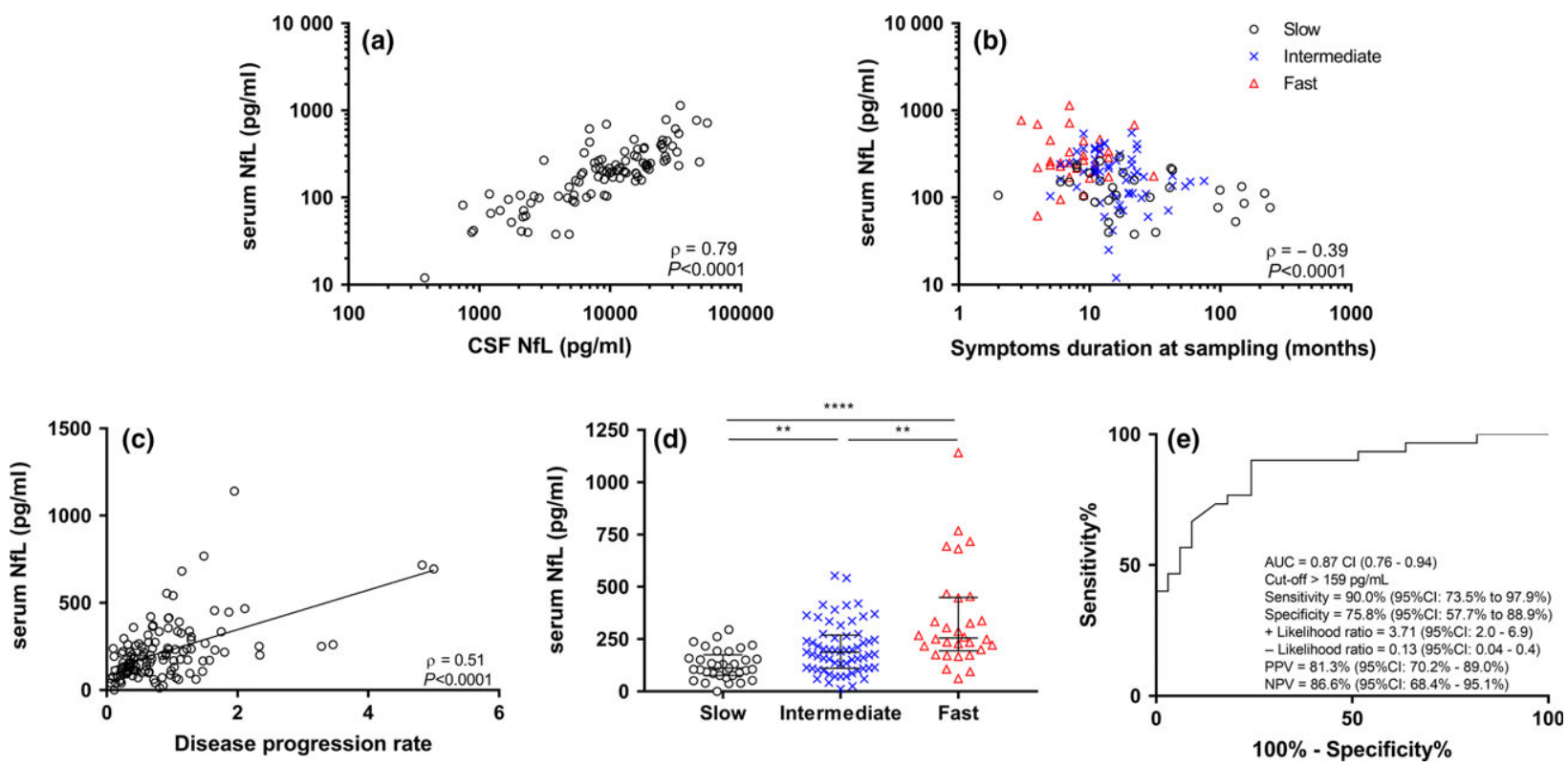

Figure 1. Correlations among the serum levels of NfL, symptom duration and the disease progression rate. (a) Correlation between the serum and CSF levels of NfL in 97 paired ALS samples (Spearman's $\rho=0.79 ; P<0.0001$ ). (b) Negative correlation between the symptom duration at blood sampling and the serum NfL levels of patients with ALS stratified according to their disease progression in slow (black circles), intermediate (blue crosses) or fast progressors (red triangles) (Spearman's $\rho=-0.39 ; P<0.0001$ ). (c) Positive correlation between the disease progression rate and the serum levels of NfL (Spearman's $\rho=0.51 ; P<0.0001$ ). (d) Serum NfL levels in patients with ALS stratified according to their disease progression rate in slow (black circles), intermediate (blue crosses) and fast progressors (red triangles). Kruskal-Wallis test corrected for multiple comparison with the Dunn's post hoc test. (e) ROC curve to discriminate patients with ALS displaying slow and fast disease progressions. ${ }^{* *} P<0.01$ and $* * * P<0.0001$ were considered as statistically significant. + Likelihood ratio, positive likelihood ratio; -Likelihood ratio, negative likelihood ratio; ALS, amyotrophic lateral sclerosis; AUC, area under the curve; CSF, cerebrospinal fluid; NfL, neurofilament light chain; ROC curve, receiver operating characteristics curve; PPV, positive predictive value; NPV, negative predictive value. 
progressors $=17.3$ months; $\quad \mathrm{IQR}=31.2 ; \quad$ range $=2.4$ 239.6 months), suggesting that the enrichment of fast disease progressors within the cohort of patients with a short disease duration from blood sampling drives the inverse correlation between NfL levels and symptom duration (Figure 1b). Serum NfL levels in patients with ALS correlated with the disease progression rate (Spearman's $\rho=0.51$, $P<0.0001$ ) (Figure 1c). The median time difference between date of ALSFRS-R scoring and date of blood sampling was 2.5 days (IQR $=25.3$ days; ranged from 0 to 90 days). Patients with ALS with a fast disease progression rate $(n=30)$ displayed significantly higher levels of serum NfL than patients with a slow $(n=33)$ (median $=255 \mathrm{pg} /$ $\mathrm{ml}$ vs. $112 \mathrm{pg} / \mathrm{ml}$, respectively, $P<0.0001)$ or intermediate disease progression rate $(n=61)(255 \mathrm{pg} / \mathrm{ml}$ vs. $188 \mathrm{pg} / \mathrm{ml}, P=0.0097$ ) (Figure $1 \mathrm{~d}$ and Table 1 ). The serum levels of NfL were also significantly higher in patients with ALS displaying an intermediate progression rate as compared with slow progressors (188 pg/ml vs. $112 \mathrm{pg} / \mathrm{ml}$, respectively, $P=0.0059$ ) (Figure $1 \mathbf{d}$ and Table 1 ). The AUC to discriminate patients between fast and slow disease progressors was 0.87 [95th percentile confidence interval (95\% CI): 0.76-0.94; $P<0.0001]$ at an optimal cut-off of $159 \mathrm{pg} / \mathrm{ml}$. At this cut-off, the diagnostic accuracy of the serum levels of NfL had a sensitivity of $90.0 \%$ (95\% CI: 73.5-97.9) and a specificity of 75.8\% (95\% CI: 57.7-88.9) with a +LR of 3.71 (95\% CI: 2.0-6.9), a - LR of 0.13 (95\% CI: $0.04-0.4)$, a PPV of $81.3 \%$ (95\% CI: 70.2-89.0) and a NPV of $86.6 \%$ (95\% CI: 68.4-95.1) (Figure 1e).

\section{Serum NfL as a marker of UMN degeneration in ALS}

Serum NfL in ALS was increased as a function of the number of regions affected by both UMN and LMN degeneration (1 region vs. 2 regions: $119 \mathrm{pg} / \mathrm{ml}$ vs. $232 \mathrm{pg} / \mathrm{ml}, \quad P=0.027 ; \quad 1$ region vs. 3 regions: $119 \mathrm{pg} / \mathrm{ml}$ vs. $268 \mathrm{pg} / \mathrm{ml}, P=0.0021$ ) (Figure 2a). In particular, the levels of serum NfL were significantly increased between patients with 1 or 3 regions affected by UMN degeneration (1 region vs. 3 regions: $106 \mathrm{pg} / \mathrm{ml}$ vs. $251 \mathrm{pg} / \mathrm{ml}, \quad P=0.0081$ ) (Figure 2b). However, the serum NfL levels were relatively stable with increasing number of regions affected by LMN degeneration as assessed with EMG, applying the Awaji criteria (1 vs. 2 regions: 191 pg/ml vs. 221 pg/ $\mathrm{ml}$; 1 region vs. 3 regions: $191 \mathrm{pg} / \mathrm{ml}$ vs. $225 \mathrm{pg} / \mathrm{ml}$ ) and revised El Escorial criteria (Figures 2c and S1, respectively). When including these parameters in an analysis of covariance together with the disease progression rate, the age at blood sampling, gender, the site of onset and the symptom duration at blood sampling as covariates, serum NfL was still elevated in patients with ALS displaying 3 regions with UMN degeneration compared with patients with ALS with only one region with UMN degeneration (adjusted means $\log 10$ serum NfL; 1 vs. 3 regions: 2.063 vs. 2.348, $P=0.0062$ ). Based on a heat map analysis, the serum levels of NfL increase together with the number of regions with UMN degeneration and the disease progression rate (Figure 3a). The levels of serum NfL increase with the disease progression rate, with the former less dependent on the number of regions affected with LMN degeneration (Figure 3b). The serum levels of NfL correlated with the disease progression rate when 1 or 2 regions are affected by UMN and LMN degeneration but not when 3 regions are affected by both UMN and LMN degeneration (Figure $3 c)$.

\section{The serum levels of NfL are elevated in patients with ALS}

The serum NfL levels were about 6 times higher in serum of patients with ALS than in patients with an ALS mimic (median serum NfL levels $179 \mathrm{pg} / \mathrm{ml}$ vs. $29 \mathrm{pg} / \mathrm{ml} ; \quad P<0.0001$ ) (Figure $4 \mathbf{a})$. The associated AUC was 0.85 (95\% CI: 0.79-0.90; $P<0.0001)$, generating an optimal cut-off of $93 \mathrm{pg} / \mathrm{ml}$ resulting in a sensitivity of $79.2 \%(95 \%$ CI: $71.8-85.4)$ and a specificity of $84.2 \%$ (95\% CI: 60.4-96.6), with a +LR of 5.02 (95\% CI: 1.8-14.2), a -LR of 0.25 (95\% CI: 0.2-0.4), a PPV of 97.5\% (95\% CI: 93.3-99.1) and a NPV of $34.0 \%$ (95\% CI: 26.3-42.7) (Figure 4b). At a fixed sensitivity of $95 \%$, the serum levels of NfL had a specificity of $52.6 \%(95 \% \mathrm{CI}: 5.3-79.0)$ to discriminate patients with ALS from ALS mimic patients. At a fixed specificity of $95 \%$ the serum levels of $\mathrm{NfL}$ had a sensitivity of $0.67 \% \quad(95 \% \mathrm{CI}$ : 0.0-61.1). As ALS is commonly associated in the differential diagnosis of patients with a cervical spondylotic myelopathy, we retrospectively retrieved an additional set of 16 serum samples of patients with a cervical stenosis/myelopathy. These patients, however, did not present as a genuine ALS mimic at our NMRC. The vast majority of these patients suffered 

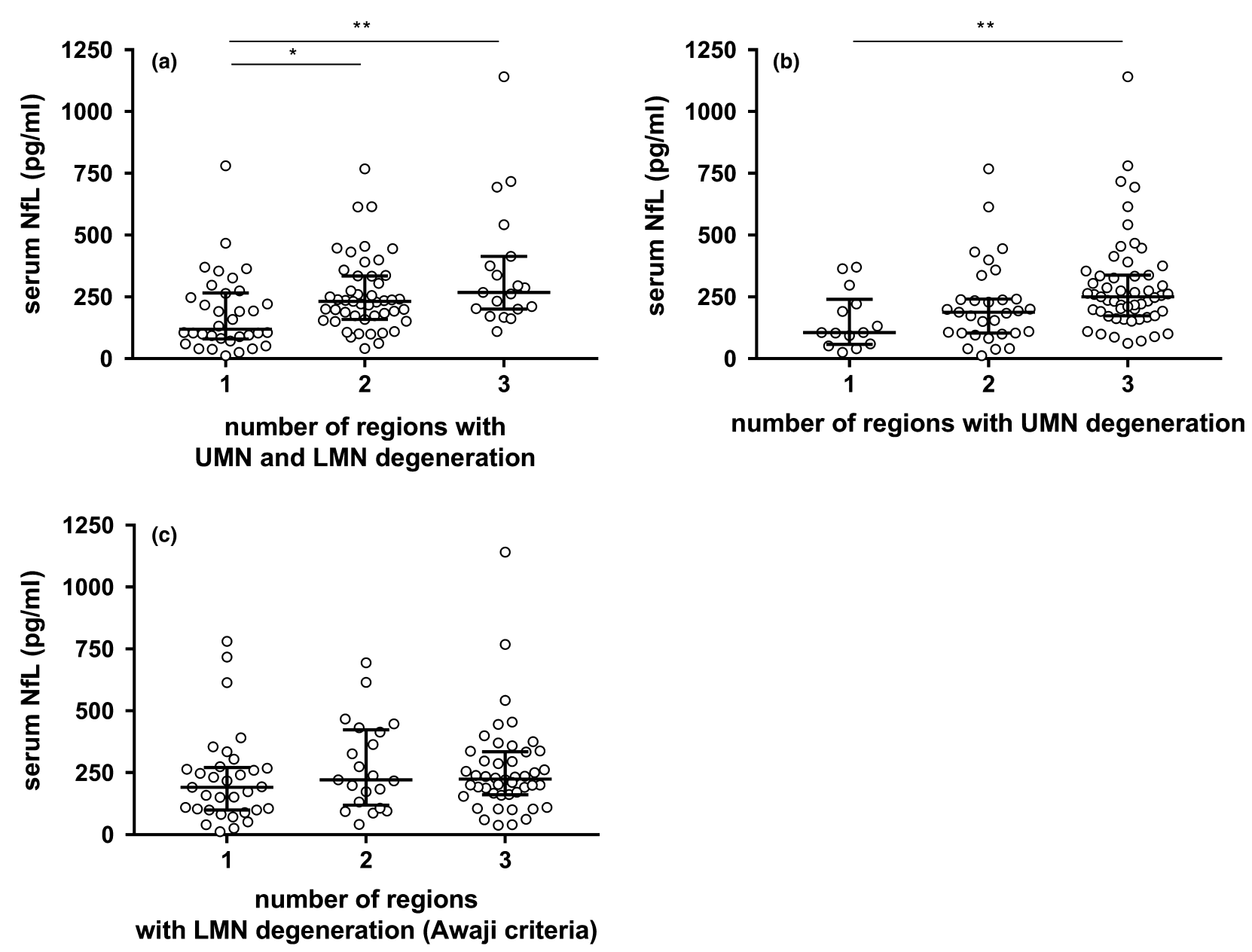

Figure 2. Correlation between the serum levels of NfL and the extent of motor neuron degeneration. (a) Comparison between the serum NfL levels in patients with ALS according to the number of regions with both UMN and LMN degeneration. (b) Comparison between the serum NfL levels in patients with ALS according to the number of regions with UMN degeneration only. (c) Comparison between the serum NfL levels in patients with ALS according to the number of regions with LMN degeneration only according to the Awaji criteria. Kruskal-Wallis corrected for multiple comparison with the Dunn's post hoc test. ALS, amyotrophic lateral sclerosis; LMN, lower motor neuron; NfL, neurofilament light chain; UMN, upper motor neuron.

from a moderate-to-severe cervical myelopathy, with among them high levels of serum NfL (Figure S2). However, the patient with cervical stenosis mimicking ALS presented with a cervical herniated disc (C5-C6) without a clear documented myelopathy, having low levels of serum NfL (Table 2).

The serum levels of NfL were significantly higher in patients with ALS as compared with patients diagnosed with PLS but not with patients with PMA (median serum NfL levels $179 \mathrm{pg} / \mathrm{ml}$ vs. $34 \mathrm{pg} / \mathrm{ml}(P=0.0008)$ and $179 \mathrm{pg} / \mathrm{ml}$ vs. $71 \mathrm{pg} / \mathrm{ml}$, respectively) (Figure 4a). However, variable levels of serum NfL were observed (range $=13-1185 \mathrm{pg} / \mathrm{ml}$ ) in patients with PMA. The serum NfL levels correlated with the disease progression rate in patients with PMA but not in patients with PLS (Spearman's $\quad \rho=0.94, \quad P=0.017 ; \quad$ Spearman's $\rho=0.32, \quad P=0.56$ ) (Table S1 and Figure S3). The serum levels of NfL in patients with ALS were significantly higher as compared to HSP, but not to GBS or CIDP (median serum NfL levels $179 \mathrm{pg} / \mathrm{ml}$ vs. $37 \mathrm{pg} /$ $\mathrm{ml}, \quad P=0.0002 ; 179 \mathrm{pg} / \mathrm{ml}$ vs. $123 \mathrm{pg} / \mathrm{ml}$ and vs. $101 \mathrm{pg} / \mathrm{ml}$, respectively) (Figure $4 \mathbf{a}$ ), which confirms that serum NfL is not specific to ALS. Indeed, ROC curve analysis revealed an AUC of 0.58 (95\% CI: $0.51-0.64, \quad P=0.097)$, at an optimal cut-off of $139 \mathrm{pg} / \mathrm{ml}$, between patients with ALS and disease 


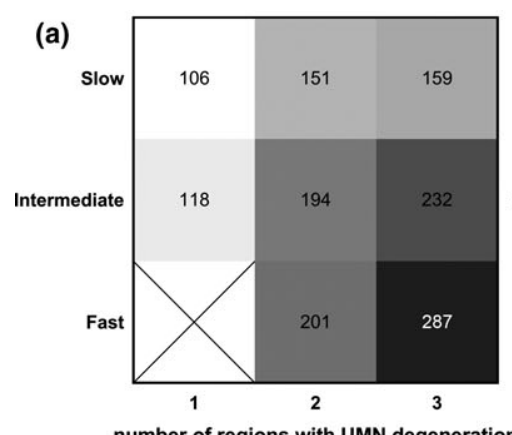

number of regions with UMN degeneration

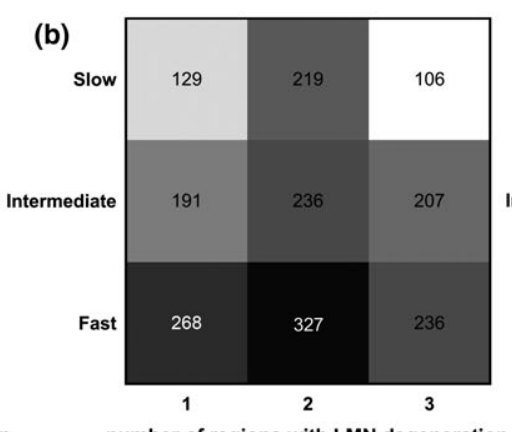

number of regions with LMN degeneration

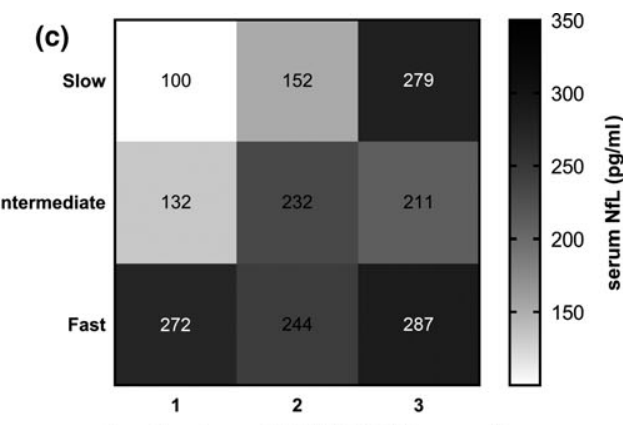

number of regions with UMN+LMN degeneration

Figure 3. Heat map of the correlations among the disease progression rate, the extent of motor neuron degeneration and the serum levels of NfL. (a) Heat map among the disease progression rate, the number of regions with UMN degeneration and the serum levels of NfL. (b) Heat map among the disease progression rate, the number of regions with LMN degeneration (Awaji criteria) and the serum levels of NfL. (c) Heat map among the disease progression rate, the number of regions displaying both UMN and LMN degeneration and the serum NfL levels. Cell labels are expressed in $\mathrm{pg} / \mathrm{ml}$ and represent the median for a given combination. The colour gradient corresponds to the median levels of serum NfL, the darker the colour gets, the higher the levels of serum NfL are. A cross ((a) fast progressor with 1 region with UMN degeneration) indicates that no patients with ALS had this combination. LMN, lower motor neuron; NfL, neurofilament light chain; UMN, upper motor neuron.
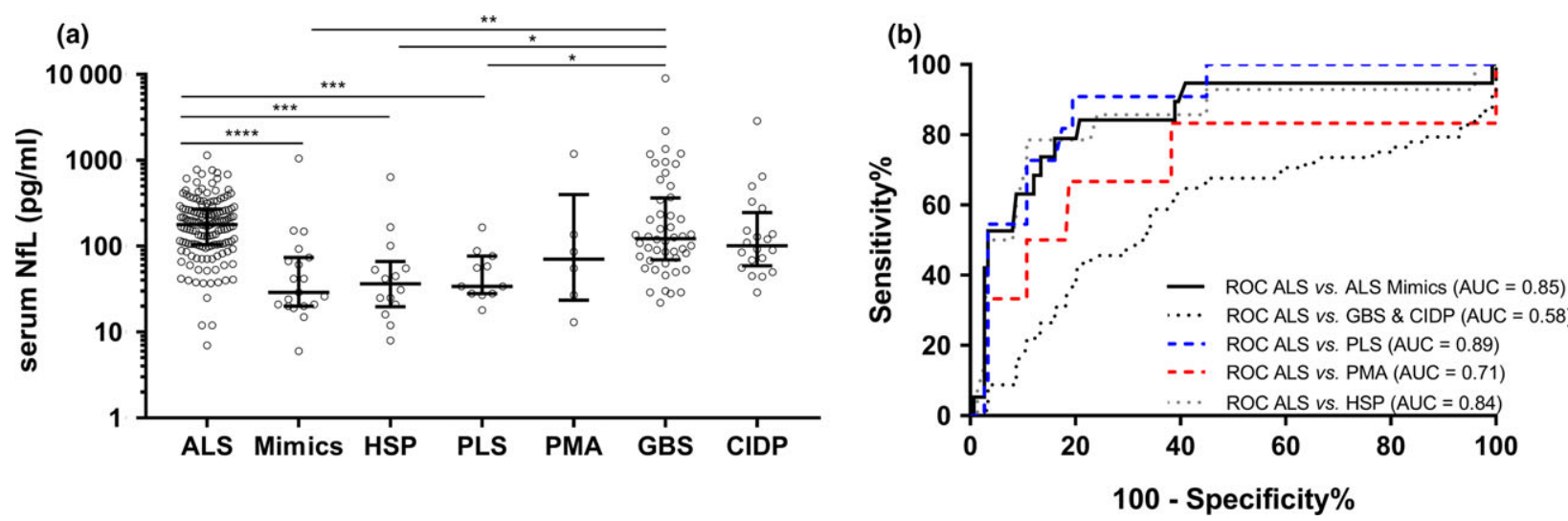

Figure 4. Serum levels of NfL in patients with ALS, ALS mimics and controls. (a) Scatter plots of the serum NfL levels in patients with ALS, disease mimics and in disease controls (median serum NfL levels; ALS vs. ALS Mimics: 179 pg/ml vs. 29 pg/ml P $<0.0001$; ALS vs. PLS: $179 \mathrm{pg} / \mathrm{ml}$ vs. $34 \mathrm{pg} / \mathrm{ml} P=0.0008$; ALS vs. HSP: $179 \mathrm{pg} / \mathrm{ml}$ vs. $37 \mathrm{pg} / \mathrm{ml} P=0.0002$; GBS vs. ALS mimics: $123 \mathrm{pg} / \mathrm{ml}$ vs. $29 \mathrm{pg} /$ $\mathrm{ml} P=0.0016$; GBS vs. PLS: $123 \mathrm{pg} / \mathrm{ml}$ vs. $34 \mathrm{pg} / \mathrm{ml} P=0.022$; GBS vs. HSP: $123 \mathrm{pg} / \mathrm{ml}$ vs. $37 \mathrm{pg} / \mathrm{ml} P=0.011)$. Kruskal-Wallis corrected for multiple comparison with the Dunn's post hoc test. (b) ROC curves to distinguish between patients with ALS vs. ALS mimics (solid black line; AUC $=0.85, P<0.0001$ ), vs. GBS and CIDP (dotted black line; AUC $=0.58, P=0.097$ ), vs. PLS (dashed blue line; AUC $=0.89, P<0.0001)$, vs. PMA (dashed red line; AUC $=0.71, P=0.13)$ and vs. HSP (dotted grey line: AUC $=0.84, P<0.0001$ ). ${ }^{*} P<0.05,{ }^{* *} P<0.01,{ }^{* * *} P<0.001$ and ${ }^{* * * * P}<0.0001$ were considered as statistically significant. ALS, amyotrophic lateral sclerosis; AUC, area under the curve; CIDP, chronic inflammatory demyelinating polyneuropathy; GBS, Guillain-Barré syndrome; HSP, hereditary spastic paraplegia; NfL, neurofilament light chain; PLS, primary lateral sclerosis; PMA, progressive muscular atrophy; ROC curve, receiver operating characteristic curve.

controls (GBS and CIDP) with a poor specificity of 63.2\% (95\% CI: 50.7-74.6) (Figure 4b). The AUC to discriminate between patients with ALS and with HSP was 0.84 (95\% CI: $0.78-0.90 ; P<0.0001)$ at an optimal cut-off of $55 \mathrm{pg} / \mathrm{ml}$, with a sensitivity of $89.3 \%$ (95\% CI: 83.1-93.7), a specificity of $78.6 \%$ (95\% CI: 49.2-95.3), a +LR of 4.2 (95\% CI: 1.5-11.4) and a
-LR of 0.14 (95\% CI: 0.1-0.2) (Figure 4b). The AUC to discriminate patients with ALS with patients with PLS was 0.89 (95\% CI: 0.83-0.93, $P<0.0001)$, at an optimal cut-off of $88 \mathrm{pg} / \mathrm{ml}$, resulting to a sensitivity of $80.5 \%$ (95\% CI: 73.3-86.6), a specificity of $90.9 \%$ (95\% CI: $58.7-99.8)$, a +LR of 8.9 (95\% CI: $1.4-57.5$ ) and a - LR of 0.2 (95\% CI: $0.1-0.3)$ (Figure $4 \mathbf{b})$. The 
AUC to distinguish patients with ALS and patients with PMA was 0.71 (95\% CI: 0.63-0.78, $P=0.13)$, at an optimal cut-off of $86 \mathrm{pg} / \mathrm{ml}$, resulting to a sensitivity of $81.2 \%$ (95\% CI: 74.0-87.1), a specificity of $66.7 \%$ (95\% CI: 22.3-95.7), a +LR of 2.4 (95\% CI: $0.8-7.6)$ and a - LR of 0.3 (95\% CI: 0.1-0.5) (Figure 4B).

\section{Serum levels of NfL are stable at follow-up}

In a subgroup of 16 patients with ALS, longitudinal samples were available. The serum levels of NfL remained relatively stable over time, although changes (mainly an increase) could be seen especially within the first 20 months after onset of disease, irrespective of the disease progression rate (Figure S4).

\section{Serum NfL levels are associated with survival}

Based on a Kaplan-Meier analysis, the survival curve of patients with ALS belonging to the different tertiles of serum NfL levels was significantly different (log-rank test $P<0.0001)$. Univariate Cox proportional-hazards regressions identified serum NfL, the disease progression rate, the number of regions with LMN degeneration, the number of regions with both UMN and LMN degeneration, the diagnostic delay, the FVC and FTD as predictors of the mortality hazard ratio (HR) in patients with ALS (Table 3). All the above-mentioned parameters were entered into a multivariate Cox model, except the number of regions with LMN degeneration to avoid circularity with the number of regions with UMN and LMN degeneration. Gender, the C9orf72 status and age were also entered into the multivariate model. On a total of 52 patients with ALS entered into the multivariate Cox regression model, 26 patients reached the end-point (death) while 26 patients did not (censored values). Among the tested covariates the serum NfL levels, the site of onset and the FVC were significantly associated with survival (Table 3). Serum NfL levels in the mid and high tertile were associated with an increased HR of 4.5 and 5.3 as compared to patients with ALS having NfL levels in the lowest tertile [HR $=4.47$ (95\% CI: $1.08-$ 18.63), $P=0.04 ; \mathrm{HR}=5.34$ (95\% CI: 1.39-20.56), $P=0.015]$ (Table 3 and Figure 5). Patients with ALS with a spinal onset were associated with an increased HR of 3.7 times compared with patients with a bulbar onset $[\mathrm{HR}=3.73$ (95\% CI: 1.16-12.01), $P=0.027]$ (Table 3). Higher score at the FVC was strongly associated with a better prognosis in patients with ALS $[\mathrm{HR}=0.97$ (95\% CI: 0.95-0.99), $P=0.005]$ (Table 3).

\section{Discussion}

In this study, we described a strong correlation between CSF and serum NfL levels in patients with ALS in line with previous reports on among others Alzheimer's disease and multiple sclerosis $[16,17]$. The strong relationship between the CSF and serum levels of NfL supports the theory of a common source for NfL originating from the central nervous system (CNS) $[16,18]$. We also showed that serum NfL levels rise as a function of the number of regions affected by UMN degeneration. This is in agreement with our previous findings where we observed a better correlation of the CSF levels of NfL with the extent of UMN degeneration than with the extent of LMN degeneration [6]. In line with this, the serum NfL levels were lower in the majority of patients with PMA, as reported before [19]. Importantly, our findings show that the serum levels of NfL increase with the extent of UMN degeneration when correcting for the disease progression rate, the site of onset, the age at blood sampling, gender or the symptom duration at time of blood sampling.

The elevation of NfL is not specific to motor neuron degeneration, as there is evidence that the source of serum NfL also originate from cortical and spinal neuronal damages $[16,18]$. In a mouse model where neuronal injury was induced by cranial irradiation, increase in serum NfL was observed, independently of the blood-brain barrier permeability [20]. The CNS origin of elevated levels of NfL in CSF and in serum is further confirmed by the correlation between rising levels of serum and CSF NfL with activation of neurodegeneration in an inducible mouse model of neurodegeneration [21]. Furthermore, elevated levels of serum and CSF NfL have been reported in other neurodegenerative diseases mainly affecting the cerebral cortex such as Alzheimer's disease [16], FTD [22] and Huntington's disease [23]. Intriguingly, it is not clear yet how NfL is shed into the peripheral blood in ALS. Brureau and colleagues have identified in CSF of Thy-Tau22 mice a $10-\mathrm{kDa}$ fragment of NfL but not the full-length NfL that is detected with a commercial enzyme-linked immunosorbent assay using the same pair of antibodies as in our study [21]. This might suggest that studies in human body fluids also detect short fragments of NfL 
Table 3. Univariate and multivariate Cox regression analysis of possible clinical predictors of survival in patients with ALS

\begin{tabular}{|c|c|c|c|c|c|c|}
\hline & & & \multicolumn{2}{|c|}{ Univariate Cox regression } & \multicolumn{2}{|c|}{$\begin{array}{l}\text { Multivariate Cox regression } \\
(n=52)\end{array}$} \\
\hline & & & HR $(95 \%$ CI) & $P$-value & HR $(95 \% C I)$ & $P$-value \\
\hline \multirow[t]{3}{*}{ Serum NfL $\dagger+$} & $n=113^{\S}$ & Low tertile & Ref & - & Ref & - \\
\hline & & Mid tertile & $6.92(2.31-20.73)$ & 0.001 & $4.47(1.08-18.63)$ & 0.04 \\
\hline & & High tertile & $10.49(3.57-30.88)$ & $<0.0001$ & $5.34(1.39-20.56)$ & 0.015 \\
\hline \multirow[t]{3}{*}{ Disease progression rate $t+$} & $n=106$ & Slow & Ref & - & Ref & - \\
\hline & & Intermediate & $3.38(1.16-9.82)$ & 0.026 & $1.24(0.30-5.17)$ & 0.77 \\
\hline & & Fast & $9.51(3.19-28.37)$ & $<0.0001$ & $1.05(0.20-5.63)$ & 0.95 \\
\hline \multirow[t]{3}{*}{$\mathrm{UMN} \dagger$} & $n=106$ & 1 Region & Ref & - & & \\
\hline & & 2 Regions & $0.47(0.19-1.20)$ & 0.113 & & \\
\hline & & 3 Regions & $1.21(0.55-2.68)$ & 0.637 & & \\
\hline \multirow{3}{*}{$\mathrm{LMN} \dagger$} & $n=106$ & 1 Region & Ref & - & & \\
\hline & & 2 Regions & $2.15(0.82-5.66)$ & 0.12 & & \\
\hline & & 3 Regions & $2.73(1.19-6.28)$ & 0.018 & & \\
\hline \multirow{3}{*}{$\mathrm{UMN}+\mathrm{LMN}+\dagger$} & $n=106$ & 1 Region & Ref & - & Ref & - \\
\hline & & 2 Regions & $1.34(0.65-2.77)$ & 0.424 & $0.94(0.26-3.42)$ & 0.93 \\
\hline & & 3 Regions & $4.51(2.02-10.09)$ & $<0.0001$ & $2.38(0.73-7.81)$ & 0.15 \\
\hline Diagnostic delay $\ddagger$ & $n=106$ & Continuous & $0.95(0.91-0.996)$ & 0.035 & $0.92(0.85-1.01)$ & 0.067 \\
\hline Forced vital capacity $\ddagger$ & $n=102$ & Continuous & $0.97(0.96-0.98)$ & $<0.0001$ & 0.97 (0.95-0.99) & 0.005 \\
\hline \multirow[t]{2}{*}{ C9orf72 statust+ } & $n=105$ & Noncarriers & Ref & - & Ref & - \\
\hline & & Carriers & $2.0(0.88-4.52)$ & 0.097 & $0.50(0.12-2.05)$ & 0.34 \\
\hline \multirow[t]{2}{*}{ FTD } & $n=113$ & Non-FTD & Ref & - & Ref & - \\
\hline & & FTD & $2.34(1.04-5.26)$ & 0.039 & $1.04(0.23-4.77)$ & 0.96 \\
\hline Age at onset & $n=107$ & Continuous & $1.01(0.99-1.04)$ & 0.307 & & \\
\hline Age at sampling $\ddagger$ & $n=113$ & Continuous & $1.01(0.99-1.04)$ & 0.408 & $1.01(0.96-1.06)$ & 0.72 \\
\hline \multirow[t]{2}{*}{ Site of onset ++9} & $n=104$ & Bulbar & Ref & - & Ref & - \\
\hline & & Spinal & $0.64(0.34-1.20)$ & 0.161 & $3.73(1.16-12.01)$ & 0.027 \\
\hline \multirow{2}{*}{ Gendert† } & $n=113$ & Male & Ref & - & Ref & \\
\hline & & Female & $1.18(0.65-2.17)$ & 0.59 & $0.82(0.28-2.42)$ & 0.73 \\
\hline
\end{tabular}

95\% CI, 95th percentile confidence interval; ALS, amyotrophic lateral sclerosis; FTD, frontotemporal dementia; HR, hazard ratio; LMN, lower motor neuron; NfL, neurofilament light chain; Ref, reference; UMN, upper motor neuron.

†Univariate analysis based on categorical values.

$\$$ Predictors entered into the multivariate model.

$\S$ A total of 36 patients with ALS were excluded from the survival analysis because possible follow-up was shorter than 1 year (Samples from December 2016 and December 2017). Univariate and Multivariate Cox regressions were performed with the backward method with a threshold to inclusion in the model of $P<0.157$.

ๆOnly spinal and bulbar onset were entered in the univariate and multivariate analysis.

that might give clues on which mechanisms are involved in the shedding of NfL from brain into blood.

Our findings are also in line with the correlation between serum NfL and the number of regions displaying a pathological brisk reflex and with white matter fibres degeneration of the superior longitudinal fasciculi and cortical spinal tract [24]. This might indicate that NfL is shed upon damage of long axons of the UMN. This is further supported by the rise of serum and CSF NfL in other diseases damaging the central nerve axons such as multiple sclerosis [25,26].

Intriguingly, serum NfL was relatively low in patients with PLS and HSP, which are two UMN syndromes. The low levels of serum NfL in PLS patients are in agreement with previous findings [8]. A reason for this lack of serum NfL elevation may be the slow speed of UMN degeneration in these disorders. In ALS, we confirmed that serum NfL was correlated with the disease progression rate, as shown previously [27]. Furthermore, the serum NfL of patients with PMA positively correlated with the disease progression rate, and a tendency was seen for patients with PLS, which requires to be confirmed on larger cohorts of patients with PLS and PMA. The relatively high levels of serum NfL in GBS and CIDP, both immune-mediated demyelinating disorders, confirmed the higher levels of NfL obtained in the CSF of 


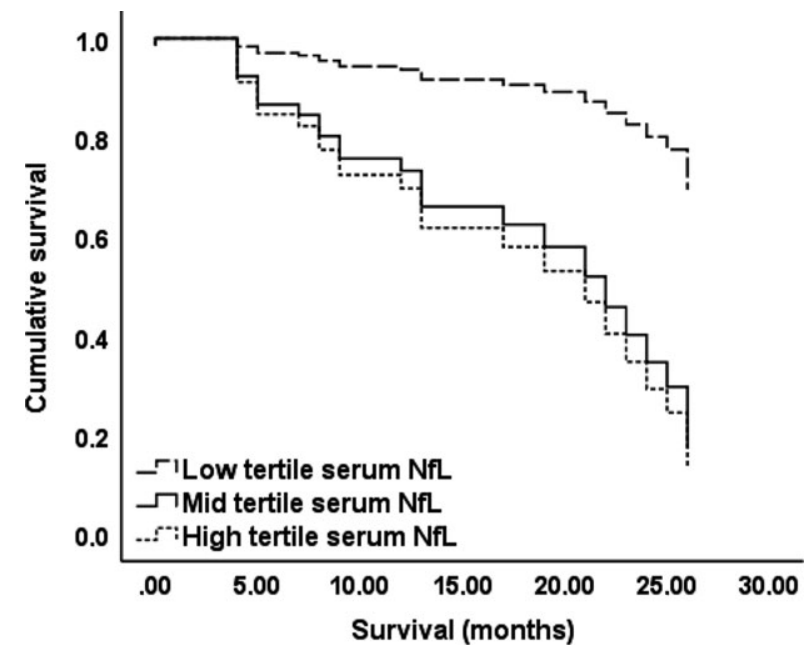

Figure 5. Multivariate Cox proportional-hazards regression survival curves of the patients with ALS according to their serum NfL levels. The serum NfL levels were stratified as low tertile $(<115 \mathrm{pg} / \mathrm{ml})$, mid-tertile $(115 \mathrm{pg} / \mathrm{ml} \leq x \leq 235 \mathrm{pg} / \mathrm{ml})$ and high tertile $(>235 \mathrm{pg} / \mathrm{ml})$. The disease progression, the tertile levels of serum NfL, the number of regions with UMN and LMN degeneration, the diagnostic delay, the age at sampling, the FVC score, the site of onset, the C9orf72 status, FTD and gender were included in the multivariate Cox model. Patients with ALS displaying a time difference between blood sampling and ALSFRS$\mathrm{R}$ or EMG or FVC $>3$ months and a follow-up between blood sampling and date of analysis of $<1$ year were excluded (excluding sampling between December 2016 and 2017). ALS, amyotrophic lateral sclerosis; ALSFRS-R, ALS functional rating scale revised; EMG, electromyography; FTD, frontotemporal dementia; FVC, forced vital capacity; LMN, lower motor neuron; NfL, neurofilament light chain; UMN, upper motor neuron.

these disorders [6,11]. Interestingly, Gaiottino and colleagues showed that the levels of serum and CSF NfL correlate in patients with GBS, suggesting a CNS origin. However, the correlation was less strong in patients with GBS than observed in patients with ALS, suggesting that serum NfL may also partly reflect demyelinating processes of the peripheral nerves in GBS [11].

Altogether, these findings are complementary and support the idea that both pNfH and NfL reflect distinct pathophysiological processes in ALS, with serum and CSF NfL levels rather reflecting UMN degeneration while CSF levels of pNfH are more specific to the ALS pathology. This is supported by our findings confirming that a rise in serum NfL is also seen in other diseases affecting the brain, and by the fact that CSF NfL, unlike CSF pNfH, is a less specific diagnostic biomarker for ALS [6]. Nevertheless, we now showed that the serum NfL levels discriminate patients with ALS and ALS mimics with a PPV of $97.5 \%$, a NPV of $34 \%$, a +LR of 5.02 and a - LR of 0.25 .

We further showed that the serum NfL levels are significantly decreased with symptoms duration to the same extent than serum pNfH levels are, as recently described [7]. This decrease, observed in a cross-sectional measurement design is driven by an enrichment of fast progressors that were sampled much earlier in the disease continuum as compared with the slow progressors. Indeed, we described that, longitudinally, the serum levels of NfL levels are relatively stable or slightly increased in a subset of patients with ALS. These results are in accordance with previous reported results for NfL in CSF and serum $[6,27,28]$. It is of interest to explore whether longitudinal measurements of serum NfL might predict onset of disease in presymptomatic individuals, including asymptomatic carriers of a hexanucleotide repeat expansion in the C9orf72 gene.

Based on univariate Cox proportional-hazards regression we showed that the disease progression rate, the serum NfL levels, the number of regions with LMN degeneration, the number of regions with UMN and LMN degeneration, the diagnostic delay, the FVC and FTD were associated with an increased HR in patients with ALS. Notably, when investigating the levels of serum NfL, independently to the other clinical parameters, patients with serum NfL levels in the highest tertile had a HR rate to mortality 10 times greater than patients with serum NfL levels in the lowest tertile.

Furthermore, in a multivariate model, only the serum NfL levels, the site of onset and the FVC were identified as significant independent predictors of survival in patients with ALS when entered together with the other covariates. Our findings confirm the previous findings that serum NfL at time of diagnosis is a strong predictor of survival [27].

Our study has some limitations: (i) We did not investigate the correlation between the pattern of white matter degeneration in patients with ALS and PLS with the serum levels of NfL. Further studies investigating the relationship between blood and CSF levels of neurofilaments (both for $\mathrm{pNfH}$ and for NfL) and brain neurodegeneration (white matter and/or gray matter degeneration) would give further valuable insights on the pathophysiological processes reflected by the neurofilaments. (ii) The low serum NfL levels detected in patients with PLS would need to be confirmed on a larger cohort to validate the involvement of the disease 
progression rate on the serum concentrations of NfL in patients with PLS. (iii) Follow-up assessments of the serum levels of NfL of asymptomatic ALS mutation carriers would give further insight on the dynamics of the neurofilament prior to symptom onset. (iv) The survival was not controlled for possible comorbidities that could influence the survival after sampling.

\section{Conclusion}

In this study, we showed that serum NfL levels were increased as a function of the disease progression rate in ALS. We also described that serum NfL correlates with the extent of motor neuron degeneration mainly driven by UMN degeneration. In contrast with the disease progression rate and the extent of motor neuron disease, the serum NfL levels were found to be strong independent predictors of survival. Our findings warrant confirmation by large multicentre studies especially with respect to increase in the number of ALS mimics, including patients with cervical stenosis/myelopathy mimicking ALS.

\section{Acknowledgements}

We would like to thank $\mathrm{PhD}$ Jens Kuhle for developing and sharing the serum NfL Meso Scale assay. The work has been supported by grants from Opening the Future Fund (KU Leuven), the Fund for Scientific Research Flanders (FWO-Flanders), the Interuniversity Attraction Poles (IUAP) programme P7/16 of the Belgian Federal Science Policy Office, the ALS Liga Belgium, the Flemish Government initiated Flanders Impulse Program on Networks for Dementia Research (VIND), the European Union Joint Programme-Neurodegenerative Disease Research (JPND) project STRENGTH and RiMod-FTD, the European E-Rare-2 project PYRAMID. PVD holds a senior clinical investigatorship of FWO-Vlaanderen and is supported by the ALS Liga Belgium and the ALS funds 'Een hart voor ALS' and 'Laeversfonds voor ALS onderzoek'. EO was supported by an ECTRIMS Postdoctoral Fellowship and AG was supported by the Research Fund KU Leuven (C24/ 16/045). This study was approved by the Ethical Committee of the University Hospitals of Leuven.

\section{Author contributions}

Concept and design of the study: BG, PVD and KP; Drafting the manuscript: BG, PVD and KP; (C) 2018 British Neuropathological Society
Interpretation and statistical analysis: BG, PVD, MDS and KP; All co-authors revised the manuscript for intellectual content.

\section{Data accessibility}

The data are available from the corresponding author on reasonable request.

\section{Conflict of interest}

None declared.

\section{References}

1 Brown RH, Al-Chalabi A. Amyotrophic lateral sclerosis. N Engl J Med 2017; 377: 162-72

2 Al-Chalabi A, Hardiman O, Kiernan MC, Chiò A, RixBrooks B, van den Berg LH. Amyotrophic lateral sclerosis: moving towards a new classification system. Lancet Neurol 2016; 15: 1182-94

3 Brooks BR, Miller RG, Swash M, Munsat TL. El Escorial revisited: revised criteria for the diagnosis of amyotrophic lateral sclerosis. Amyotroph Lateral Scler Other Motor Neuron Disord 2000; 1: 293-9

4 de Carvalho M, Dengler R, Eisen A, England JD, Kaji R, Kimura J, Mills K, Mitsumoto H, Nodera H, Shefner J, Swash M. Electrodiagnostic criteria for diagnosis of ALS. Clin Neurophysiol 2008; 119: 497-503

5 Schrooten M, Smetcoren C, Robberecht W, Van Damme P. Benefit of the Awaji diagnostic algorithm for amyotrophic lateral sclerosis: a prospective study. Ann Neurol 2011; 70: 79-83

6 Poesen K, De Schaepdryver M, Stubbendorf B, Gille B, Muckova P, Wendler S, Prell T, Ringer T, Rhode H, Stevens O, Claeys KG, Couwelier G, D'Hondt A, Lamaire N, Tilkin P, Van Reijen D, Gourmaud S, Hammer N, Heiling B, Rumpel M, Schenk A, Gunkel A, Witte OW, Paquet C, Vandenberghe R, Grosskreutz J, Van Damme P. Neurofilament markers for ALS correlate with extent of upper and lower motor neuron disease. Neurology 2017; 88: 2302-9

7 De Schaepdryver M, Jeromin A, Gille B, Claeys KG, Herbst V, Brix B, Van Damme P, Poesen K. Comparison of elevated phosphorylated neurofilament heavy chains in serum and cerebrospinal fluid of patients with amyotrophic lateral sclerosis. J Neurol Neurosurg Psychiatry 2018; 89: 367-373

8 Feneberg E, Oeckl P, Steinacker P, Verde F, Barro C, Van Damme P, Gray E, Grosskreutz J, Jardel C, Kuhle J, Koerner S, Lamari F, Amador MDM, Mayer B, Morelli C, Muckova P, Petri S, Poesen K, Raaphorst J, Salachas F, Silani V, Stubendorff B, Turner MR, Verbeek 
MM, Weishaupt JH, Weydt P, Ludolph AC, Otto M. Multicenter evaluation of neurofilaments in early symptom onset amyotrophic lateral sclerosis. Neurology 2018; 90: e22-30. https://doi.org/10.1212/WNL. 0000000000004761

9 Steinacker P, Feneberg E, Weishaupt J, Brettschneider J, Tumani H, Andersen PM, von Arnim CAF, Böhm S, Kassubek J, Kubisch C, Lulé D, Müller H-P, Muche R, Pinkhardt E, Oeckl P, Rosenbohm A, Anderl-Straub S, Volk AE, Weydt P, Ludolph AC, Otto M. Neurofilaments in the diagnosis of motoneuron diseases: a prospective study on 455 patients. J Neurol Neurosurg Psychiatry 2016; 87: 12-20

10 De Carvalho M, Swash M. Awaji diagnostic algorithm increases sensitivity of El Escorial criteria for ALS diagnosis. Amyotroph Lateral Scler 2009; 10: 53-7

11 Gaiottino J, Norgren N, Dobson R, Topping J, Nissim A, Malaspina A, Bestwick JP, Monsch AU, Regeniter A, Lindberg RL, Kappos L, Leppert D, Petzold A, Giovannoni G, Kuhle J. Increased neurofilament light chain blood levels in neurodegenerative neurological diseases. PLoS ONE 2013; 8: e75091

12 Delva A, Thakore N, Pioro EP, Poesen K, Saunders-Pullman R, Meijer IA, Rucker JC, Kissel JT, Van Damme P. Finger extension weakness and downbeat nystagmus motor neuron disease syndrome: A novel motor neuron disorder? Muscle Nerve 2017; 56: 1164-8

13 Gomeni R, Fava M; Pooled Resource Open-Access ALS Clinical Trials Consortium. Amyotrophic lateral sclerosis disease progression model. Amyotroph Lateral Scler Frontotemporal Degener 2014; 15: 119-29

14 Reniers W, Schrooten M, Claeys KG, Tilkin P, D’Hondt A, Van Reijen D, Couwelier G, Lamaire N, Robberecht W, Fieuws S, Van Damme P. Prognostic value of clinical and electrodiagnostic parameters at time of diagnosis in patients with amyotrophic lateral sclerosis. Amyotroph Lateral Scler Frontotemporal Degener 2017; 18: $341-50$

15 Westeneng HJ, Debray TPA, Visser AE, van Eijk RPA, Rooney JPK, Calvo A, Martin S, McDermott CJ, Thompson AG, Pinto S, Kobeleva X, Rosenbohm A, Stubendorff B, Sommer H, Middelkoop BM, Dekker AM, van Vugt JJFA, van Rheenen W, Vajda A, Heverin M, Kazoka M, Hollinger H, Gromicho M, Körner S, Ringer TM, Rödiger A, Gunkel A, Shaw CE, Bredenoord AL, van Es MA, Corcia P, Couratier P, Weber M, Grosskreutz J, Ludolph AC, Petri S, de Carvalho M, Van Damme P, Talbot K, Turner MR, Shaw PJ, Al-Chalabi A, Chiò A, Hardiman O, Moons KGM, Veldink JH, van den Berg LH. Prognosis for patients with amyotrophic lateral sclerosis: development and validation of a personalised prediction model. Lancet Neurol 2018; 17: 423-33

16 Mattsson N, Andreasson U, Zetterberg H, Blennow K. Association of plasma neurofilament light with neurodegeneration in patients with alzheimer disease. JAMA Neurol 2017; 74: 557-66
17 Piehl F, Kockum I, Khademi M, Blennow K, Lycke J, Zetterberg $\mathrm{H}$, Olsson T. Plasma neurofilament light chain levels in patients with MS switching from injectable therapies to fingolimod. Mult Scler 2017; 24: 1046-1054

18 Kuhle J, Gaiottino J, Leppert D, Petzold A, Bestwick JP, Malaspina A, Lu CH, Dobson R, Disanto G, Norgren N, Nissim A, Kappos L, Hurlbert J, Yong VW, Giovannoni G, Casha S. Serum neurofilament light chain is a biomarker of human spinal cord injury severity and outcome. J Neurol Neurosurg Psychiatry 2015; 86: 273-9

19 Gaiani A, Martinelli I, Bello L, Querin G, Puthenparampil M, Ruggero S, Toffanin E, Cagnin A, Briani C, Pegoraro E, Sorarù G. Diagnostic and prognostic biomarkers in amyotrophic lateral sclerosis. JAMA Neurol 2017; 74: 525532

20 Kalm M, Boström M, Sandelius Å, Eriksson Y, Ek CJ, Blennow K, Björk-Eriksson T, Zetterberg H. Serum concentrations of the axonal injury marker neurofilament light protein are not influenced by blood-brain barrier permeability. Brain Res 2017; 1668: 12-19

21 Brureau A, Blanchard-Bregeon V, Pech C, Hamon S, Chaillou P, Guillemot JC, Barneoud P, Bertrand P, Pradier L, Rooney T, Schussler N. NF-L in cerebrospinal fluid and serum is a biomarker of neuronal damage in an inducible mouse model of neurodegeneration. Neurobiol Dis 2017; 104: 73-84

22 Meeter LH, Dopper EG, Jiskoot LC, Sanchez-Valle R, Graff C, Benussi L, Ghidoni R, Pijnenburg YA, Borroni B, Galimberti D, Laforce RJ, Masellis M, Vandenberghe R, Le BI, Otto M, van Minkelen R, Papma JM, Rombouts SA, Balasa M, Öijerstedt L, Jelic V, Dick KM, Cash DM, Harding SR, Jorge Cardoso M, Ourselin S, Rossor MN, Padovani A, Scarpini E, Fenoglio C, Tartaglia MC, Lamari F, Barro C, Kuhle J, Rohrer JD, Teunissen CE, van Swieten JC. Neurofilament light chain: a biomarker for genetic frontotemporal dementia. Ann Clin Transl Neurol 2016; 3: 623-36

23 Byrne LM, Rodrigues FB, Blennow K, Durr A, Leavitt BR, Roos RAC, Scahill RI, Tabrizi SJ, Zetterberg H, Langbehn D, Wild EJ. Neurofilament light protein in blood as a potential biomarker of neurodegeneration in Huntington's disease: a retrospective cohort analysis. Lancet Neurol 2017; 16: 601-9

24 Menke RAL, Gray E, Lu C-H, Kuhle J, Talbot K, Malaspina A, Turner MR. CSF neurofilament light chain reflects corticospinal tract degeneration in ALS. Ann Clin Transl Neurol 2015; 2: 748-55

25 Disanto G, Barro C, Benkert P, Naegelin Y, Schädelin S, Giardiello A, Zecca C, Blennow K, Zetterberg H, Leppert D, Kappos L, Gobbi C, Kuhle J. Serum Neurofilament light: a biomarker of neuronal damage in multiple sclerosis. Ann Neurol 2017; 81: 857-70

26 Kuhle J, Nourbakhsh B, Grant D, Morant S, Barro C, Yaldizli Ö, Pelletier D, Giovannoni G, Waubant E, Gnanapavan S. Serum neurofilament is associated 
with progression of brain atrophy and disability in early MS. Neurology 2017; 88: 826-31

$27 \mathrm{Lu} \mathrm{CH}$, Macdonald-Wallis C, Gray E, Pearce N, Petzold A, Norgren N, Giovannoni G, Fratta P, Sidle K, Fish M, Orrell R, Howard R, Talbot K, Greensmith L, Kuhle J, Turner MR, Malaspina A. Neurofilament light chain: a prognostic biomarker in amyotrophic lateral sclerosis. Neurology 2015; 84: 2247-57

28 Steinacker P, Huss A, Mayer B, Grehl T, Grosskreutz J, Borck G, Kuhle J, Lulé D, Meyer T, Oeckl P, Petri S, Weishaupt J, Ludolph AC, Otto M. Diagnostic and prognostic significance of neurofilament light chain NF-L, but not progranulin and S100B, in the course of amyotrophic lateral sclerosis: data from the German MND-net. Amyotroph Lateral Scler Frontotemporal Degener 2017; 18: 112-19

\section{Supporting information}

Additional Supporting Information may be found in the online version of this article at the publisher's web-site:

Figure S1. Comparison of the serum neurofilament light chain (NfL) levels according to the number of regions with lower motor neuron (LMN) degeneration only.

Figure S2. Samples of patients with cervical stenosis/ myelopathy that have been retrospectively retrieved from hospital's serum biobank.

Figure S3. Correlation between the disease progression rate and the serum neurofilament light chain (NfL) levels in patients with progressive muscular atrophy (PMA) and primary lateral sclerosis (PLS).

Figure S4. Longitudinal assessment of the serum levels of neurofilament light chain (NfL) as a function of time after blood sampling in patients with amyotrophic lateral sclerosis (ALS).

Table S1. Clinical parameters of the primary lateral sclerosis (PLS) and progressive muscular atrophy (PMA) cohorts.

Received 9 February 2018

Accepted after revision 24 May 2018

Published online Article Accepted on 16 June 2018 